IMA Journal of Management Mathematics Page 1 of 25 doi:10.1093/imaman/dri000

\title{
Measuring the risk of a nonlinear portfolio with fat tailed risk factors through probability conserving transformation
}

\author{
P. DATE AND R. Bustreo \\ Department of Mathematical Sciences, Brunel University, Uxbridge UB8 3PH, UK.
}

\begin{abstract}
This paper presents a new heuristic for fast approximation of VaR (Value-at-Risk) and CVaR (conditional Value-at-Risk) for financial portfolios where the net worth of portfolio is a nonlinear function of possibly non-Gaussian risk factors. The proposed method is based on mapping non-normal marginal distributions into normal distributions via a probability conserving transformation and then using a quadratic, i.e. Delta-Gamma approximation for the portfolio value. The method is extremely general and can deal with a wide range of marginal distributions of risk factors, including non-parametric distributions. Its computational load is comparable with Delta-Gamma-Normal method based on Fourier inversion. However, unlike the Delta-Gamma-Normal method, the proposed heuristic preserves the tail behavior of the individual risk factors, which may be seen as a significant advantage. We demonstrate the utility of the new method with comprehensive numerical experiments on simulated as well as real financial data.
\end{abstract}

Keywords: Value-at-Risk, Conditional Value-at-Risk, fat tailed distributions.

\section{Introduction}

All financial institutions need to perform effective risk management. Quantitative risk measures have become crucial management instruments for portfolio managers. Risk managers and market regulators have to control their risks or to appropriately allocate their capital. Value-at-Risk (VaR) has been chosen by the Basel Committee on Banking Supervision in Basel II as the standard risk measure for financial risk managers, see e.g. Basel Committee (2006) and Chen \& Gerlach (2011) for details. It measures the worst expected loss under normal market conditions over a specific time interval at a given confidence interval. It may be seen as the "best of worst cases scenario" and it therefore systematically underestimates the potential losses associated with the specified level of probability. To define VaR, we consider a real-valued random variable $\mathscr{X}$ on a probability space $(\Omega, \mathscr{A}, P)$ that expresses the random profit or loss of some asset or portfolio, its cumulative distribution function (cdf) $F_{\mathscr{X}}(x)=P(\mathscr{X} \leqslant x)$ and a confidence level $\alpha \in(0,1)$. Therefore the quantity $q_{\alpha}(\mathscr{X})=\inf \left\{x \mid F_{\mathscr{X}}(x) \geqslant \alpha\right\}$ is the $\alpha$-quantile of $\mathscr{X}$. Then VaR at level $\alpha$ is given by:

$$
\operatorname{VaR}_{\alpha}=-q_{1-\alpha}(\mathscr{X}) .
$$

VaR has received criticism by Artzner et al. (1999), Acerbi \& Tasche (2001) and Szegö (2005) for not being a coherent measure of risk. This is because of the fact that it does not generally fulfill the axiom of sub-additivity, apart from the case of linear portfolios with normally distributed risk factors and similar special cases. A sub-additive alternative to VaR is the conditional Value-at-Risk (CVaR), defined as the conditional expectation of loss for losses beyond the VaR level. CVaR and its minimization formula were first developed in Rockafellar \& Uryasev (2000). There, authors demonstrated the numerical efficiency of their proposed procedure through several case studies, including portfolio optimization and option hedging. The conditional Value-at-Risk at level $\alpha$ can be defined as:

$$
C \operatorname{VaR}_{\alpha}=-\mathbb{E}\left(\mathscr{X} \mid \mathscr{X} \leqslant q_{1-\alpha}(\mathscr{X})\right) .
$$


Computation of CVaR, in effect, requires computing VaR at several different confidence levels and hence is typically computationally more intensive.

In general, the computation of $\mathrm{VaR}$ or $\mathrm{CVaR}$ for a financial portfolio leads to one of the four possible scenarios:

1. The portfolio is linear in the underlying risk factors and the distribution of risk factors can be approximated by a Gaussian distribution. This is a case when the portfolio consists of stocks or stock futures, risk factors are stock returns only and the confidence level is $\alpha=0.95$ or lower. For higher $\alpha$ values, distributional assumption of Gaussianity is inappropriate, as has been proven time and again in literature. Nevertheless, Gaussian approach remains popular due to its simplicity and is often used as a starting point or as benchmark; see, e.g. Albanese et al. (2004) and Alexander (2008).

2. The second scenario is the one which involves a linear portfolio with non-Gaussian risk factors. This means that the distribution of the portfolio net worth may be different from that of the underlying risk factors. Typically, this scenario requires a Monte Carlo simulation to compute the quantiles of portfolio value distribution. There are two distinct choices of risk factor distributions followed in literature; the approach based on extreme value theory is followed in Embrechts et al. (1999) and Gilli \& Këllezi (2006), among others. On the other hand, different thick tailed distributions which do not belong to the class of extreme value distributions have also been used, including the use of mixture of normals in Zangari (1996) and Duffie \& Pan (1997), Student's $t$ as outlined in Alexander (2008) and the use of mixture of elliptic distributions in Kamdem (2003). An alternative approach is provided by the copula-based models (see e.g. Nelsen (2006) for an introduction to copulas) in Rahman et al. (2011) and in Sak \& Haksöz (2011), among others.

3. The third scenario is when the portfolio has Gaussian risk factors but the portfolio value is a nonlinear function of risk factors, e.g. due to presence of derivative instruments such as options. A very common way of dealing with this scenario is using a quadratic (or Delta-Gamma) approximation to the portfolio value, since quantiles of a quadratic form of jointly Gaussian random variables can be efficiently computed using Fourier inversion integral: see, e.g. Glasserman (2003) for a detailed treatment. Other approaches include Cornish-Fisher expansion as discussed in Jaschke (2001) and Zangari (1996), Johnson transformation discussed in Zangari (1996), Solomon-Stephens approximations discussed in Britten-Jones \& Schaefer (1999), saddle point approximation outlined in Feuerverger \& Wong (2000) and other Fourier inversion based methods described in Albanese et al. (2004) and Rouvinez (1997). Monte Carlo simulation with the quadratic approximation of the portfolio value (so-called partial Monte Carlo simulation) is computationally typically simpler than full Monte Carlo, as the computational load of pricing nonlinear instruments for given values of risk factors may be far greater than computing quadratic forms of risk factors; see Pritsker (1996) for an application. Comparisons of accuracy and efficiency among mentioned methodologies are carried out in Mina \& Ulmer (1999) and in Castellacci \& Siclari (2003). Different methods for dealing with this Gaussian risk factors, nonlinear portfolio scenarios are compared in Britten-Jones \& Schaefer (1999) and in Pichler \& Selitsch (1999).

4. The last and the most general scenario involves a portfolio with nonlinear instruments which depend on non-Gaussian risk factors. Any financial portfolios involving derivative instruments on stocks (such as hedge funds or absolute return funds) fall under this category. Unlike the normal 
distribution, the quadratic forms of fat-tailed distributions are not amenable to easy evaluation in general. Apart from full Monte Carlo or partial Monte Carlo simulation (using quadratic approximation of nonlinear functions), few computationally cheap alternatives currently exist for this scenario; see Glasserman et al. (2000) and El-Jahel et al. (1999) for some approaches to this problem.

The financial crisis of 2008 has brought back to researchers and managers the fact that normality assumption for risk factors is not realistic. Gaussian distribution-based models are appealing because of their simple implementation, but they fail to explain real world risk factor characteristics such as fattailedness and skewness. In Nozari et al. (2010), Sheikh \& Qiao (2010) and Stoyanov et al. (2011) authors highlight the fact that empirical research on financial returns carried out since 1950s leads to the need to consider several phenomena including fat tails, skewness and serial correlation. Therefore models that rely on the assumption of normal distributions need to be relaxed in order to identify a potential much higher level of risk. Parsimonious Gaussian-based models are often employed by practitioners despite empirical evidence to the contrary. However, especially during periods of high turbulence in the financial markets, such as the one in the recent crisis, Gaussianity assumption can lead to severe under-estimation of risk.

The focus of this paper is to provide a heuristic to calculate $\mathrm{VaR}$ and $\mathrm{CVaR}$ for the last scenario mentioned above, i.e. for nonlinear portfolios with non-normal risk factors. The proposed heuristic is computationally cheaper than a full or partial Monte Carlo with fat tailed distributions, yet has a potential to be far more accurate than normal distribution-based evaluation. The novelty of our heuristic lies in offering an alternative with a potentially intermediate level of complexity and accuracy between the two extremes: between carrying out a full Monte Carlo simulation by sampling from fat tailed distributions followed by multiple pricing function evaluations on one hand and evaluating a single, one dimensional integral assuming a normal distribution and a quadratic approximation of the portfolio value on the other hand. Our approach rests on transforming the problem with non-Gaussian marginals into Gaussian ones via a probability conserving transformation, as used in Sornette et al. (2000). Unlike mean-variance based Gaussian approximation of marginal densities, probability conserving transformation retains the tail behavior which is crucial in the computation of risk measures. This transformation is then followed by construction of a joint Gaussian density and construction of a quadratic form in the jointly Gaussian transformed variables. One can then use the Delta-Gamma-Normal method for nonlinear portfolios with Gaussian risk factors for the computation of risk measures. Further, one can re-use the existing code for the fast Fourier transform (FFT) based evaluation of CVaR using Delta-Gamma-Normal method, by using the proposed heuristic of transforming the risk factors into Gaussian factors.

The rest of the paper is structured as follows. Section 2 establishes the notation used and outlines the Delta-Gamma-Normal model for ease of reference. Section 3 introduces the probability conserving transformation which is used later on in this work. Section 4 describes the proposed method, which we refer to as Delta-Gamma- $Q$, for non-normal risk factors and nonlinear portfolios. Section 5 illustrates this method with extensive simulation experiments with nonlinear portfolios. Section 6 presents backtesting with real option price data and discusses on the advantages in computational terms of DeltaGamma-Q over partial Monte Carlo and finally section 7 concludes the article with comments on future research directions.

\section{The Delta-Gamma-Normal model}

Let us recall the model developed in Glasserman (2003) for computation of Value-at-Risk with normally distributed stock returns, but a portfolio which depends nonlinearly on stock prices. We introduce the 
following notation:

$$
\begin{aligned}
\mathscr{S} & =\text { vector of } m \text { market prices and rates; } \\
\Delta t & =\text { risk-measurement horizon; } \\
\Delta \mathscr{S} & =\text { change in } \mathscr{S} \text { over interval } \Delta t ; \\
\Pi(\mathscr{S}, t) & =\text { portfolio value at time } t \text { and market prices } \mathscr{S} ; \\
\mathscr{L} & =\text { loss over interval } \Delta t=-\Delta \Pi=\Pi(\mathscr{S}, t)-\Pi(\mathscr{S}+\Delta \mathscr{S}, t+\Delta t) ; \\
F_{\mathscr{L}}(x) & =P(\mathscr{L}<x), \text { the distribution of } \mathscr{L} .
\end{aligned}
$$

The time interval $\Delta t$ is typically very short, while the number $m$ of risk factors could reach thousands. Throughout this paper, we use 1 day as our time interval for computing VaR, although it is straightforward to generalize the results to other time horizons. A portfolio that includes options depends nonlinearly on the underlying asset prices and many fixed-income securities have a nonlinear dependence on interest rates. The change in portfolio value $\Delta \Pi(\mathscr{S}, t)$ is defined as $\Pi(\mathscr{S}+\Delta \mathscr{S}, t+\Delta t)-\Pi(\mathscr{S}, t)$. Some nonlinearity can be captured using Taylor expansion until the quadratic term:

$$
\Delta \Pi(\mathscr{S}, t) \approx \Theta \Delta t+\delta^{T} \Delta \mathscr{S}+\frac{1}{2} \Delta \mathscr{S}^{T} \Gamma \Delta \mathscr{S}
$$

where the vector $\Delta \mathscr{S}$ denotes the change of the underlying value, while the scalar $\Theta=\frac{\partial \Pi(\mathscr{S}, t)}{\partial t}$, the vector $\delta=\frac{\partial \Pi(\mathscr{S}, t)}{\partial \mathscr{S}}$ and the matrix $\Gamma=\frac{\partial^{2} \Pi(\mathscr{S}, t)}{\partial \mathscr{S}^{2}}$ represent the sensitivities of an instrument at time $t$. Given the portfolio $\Pi$, all the sensitivities $\Theta, \delta$ and $\Gamma$ are assumed to be given exogenously for the purpose of this paper. For individual derivative instruments such as options, $\delta$ values are provided by commercial economic data providers such as Bloomberg. Depending on the nature of derivative instruments in the portfolio, these sensitivity values may be found using a variety of methods from market data and from commonly used risk neutral models, including finite differences, implied volatilities, Malliavin calculus (for use of Malliavin calculus in computing sensitivities of certain exotic options, see e.g. Montero \& Kohatsu-Higa (2003)), etc; see, e.g. Glasserman (2003) and Hull (2006). Sensitivities $\delta$ and $\Gamma$ are regularly computed for hedging purposes by single trading desks and can be combined (at the end of the day, for example). This leads to a quadratic approximation of $\mathscr{L}$. Assuming $\Delta \mathscr{S} \sim \mathscr{N}\left(0, \Sigma_{\mathscr{S}}\right)$, then we can write:

$$
\Delta \mathscr{S}=C \mathscr{Z} \quad \text { with } \quad C C^{T}=\Sigma_{\mathscr{S}}
$$

where $\mathscr{Z} \sim \mathscr{N}(0, I)$ and $C$ is a square root of $\Sigma_{\mathscr{S}}$. Square root matrices are not unique and the exact choice of $C$ is decided as follows. We can re-write 2.1 in terms of $\mathscr{Z}$ :

$$
\mathscr{L} \approx a-\left(C^{T} \delta\right)^{T} \mathscr{Z}-\frac{1}{2} \mathscr{Z}^{T}\left(C^{T} \Gamma C\right) \mathscr{Z},
$$

with $a=-\Theta \Delta t$ deterministic. As shown in Glasserman (2003), a correct choice of $C$ allows us to express $\mathscr{L}$ as:

$$
\mathscr{L} \approx a+\mathbf{b}^{T} \mathscr{Z}+\mathscr{Z}^{T} \Lambda \mathscr{Z}=a+\sum_{i=1}^{m}\left(b_{i} \mathscr{Z}_{i}+\lambda_{i} \mathscr{Z}_{i}^{2}\right) \equiv \mathscr{Q}
$$

where $\mathbf{b}=-C^{T} \delta, \lambda_{i}$ are the $m$ diagonal values of the matrix $\Lambda=-\frac{1}{2} C^{T} \Gamma C$ and $C$ is a square root of $\Sigma_{\mathscr{S}}$ such as $C=\hat{C} U$, where $\hat{C}$ is the Cholesky factor of $\Sigma_{\mathscr{S}}$ and $U$ is the matrix of normalized eigenvectors of $-\frac{1}{2} \hat{C}^{T} \Gamma \hat{C}$. Since we approximated $P(\mathscr{L}>x) \approx P(\mathscr{Q}>x)$, now we have to derive the distribution of 
Q. It turns out that this can be determined by characteristic function of a quadratic form of Gaussian distribution, which has a closed form. In particular, the characteristic function of $\mathscr{Q}$ as expressed by (2.3) is

$$
\varphi_{\mathscr{Q}}(t)=\mathbb{E}\left[e^{j t \mathscr{Q}}\right]=e^{\psi(j t)}=e^{j t a} \prod_{i=1}^{m} \frac{1}{\sqrt{1-2 j \lambda_{i} t}} \exp \left(-\frac{1}{2} \sum_{i=1}^{m} \frac{b_{i}^{2} t^{2}}{1-2 j \lambda_{i} t}\right),
$$

where $j=\sqrt{-1}$. Using inversion integral (see, e.g. Paolella (2007)), one can retrieve the probability distribution function (pdf) or cdf of a continuous random variable. The characteristic function inversion using the FFT algorithm given the continuous random variable $\mathscr{Q}$, with pdf $f_{\mathscr{Q}}$ and characteristic function $\varphi_{\mathscr{Q}}$, for $l, u \in \mathbb{R}$ and $N \in \mathbb{N}$, can be carried out - as proposed by Paolella (2007) - recognizing that:

$$
\varphi_{\mathscr{Q}}(t)=\int_{-\infty}^{\infty} f_{\mathscr{Q}}(q) e^{j t q} d q \approx \int_{l}^{u} f_{\mathscr{Q}}(q) e^{j t q} d q \approx \sum_{k=0}^{N-1} f_{\mathscr{Q}}\left(q_{k}\right) \Delta q e^{j t q_{k}}
$$

where $q_{k}=l+k \Delta q$, with $k=0,1, \ldots, N-1$ and $\Delta q=(u-l) / N$. Creating a suitable grid of $r$-values one can compute the quantities:

$$
q(n) \approx \frac{1}{N} \varphi_{\mathscr{Q}}\left(r_{n}\right) e^{-j r_{n} l}
$$

and then using FFT to obtain an approximation to pdf $f_{\mathscr{Q}}\left(q_{k}\right)$ at discrete points $q_{k}$. Now, using the formulae (1.1) and (1.2) (with an approximation of the expectation integral by an appropriate summation), one can obtain VaR and CVaR. Note that there are two reasons for truncating Taylor's series after two terms. The first reason is the computational simplicity of computing quantiles of a quadratic form of a normal distribution (as outlined above). Secondly, widely reported empirical experience supports the use of second order approximation as adequate reflection of changes in portfolio value, especially over short time horizons as considered in this paper.

In Glasserman et al. (2000), the authors relax the assumption that the risk factors are normally distributed and demonstrate that the result can be extended to risk factors which have a multivariate $t$ distribution. In this paper, we go a lot further in generalizing Delta-Gamma-Normal method by using possibly non-parametric distributions. The key to our method is a probability conserving transformation, which is described next.

\section{Probability conserving transformation}

The idea of probability conserving transformation has been used in the context of analysis of portfolio strategies in Sornette et al. (2000). We employ this idea in the current context of risk computation. Suppose that we have a set of dependent random variables $\mathscr{X}_{1}, \mathscr{X}_{2}, \cdots, \mathscr{X}_{m}$ with arbitrary marginal cdfs $F_{i}, i=1,2, \cdots, m$. For each $\mathscr{X}_{i}$, samples $x_{j}^{(i)}, j=1,2, \cdots, n$ drawn from $F_{i}(x)$ are available. In the present context, $\mathscr{X}$ might represent a vector of asset returns or any other risk factors. Denoting the standard normal cdf by $\Phi(y)$, define a function of $\mathscr{X}_{i}$ by

$$
\psi\left(\mathscr{X}_{i}\right):=\Phi^{-1} F_{i}\left(\mathscr{X}_{i}\right),
$$

and let $y_{j}^{(i)}=\psi\left(x_{j}^{(i)}\right)$. If we envisage $y_{j}^{(i)}$ to be a sample of a random variable $\mathscr{Y}_{i}$ with standard normal distribution, then it is clear that $\Phi\left(y_{j}^{(i)}\right)=F_{i}\left(x_{j}^{(i)}\right) . \psi$ is the so-called probability conserving transformation. We can then find the sample covariance matrix of $\mathscr{Y}$ using $y_{j}^{(i)}$. We don't know a priori the joint distribution of the vector of transformed vector-valued random variable, $\mathscr{Y}$. However we can introduce 
an approximation using a standard result from information theory (Rao (2001)): conditioned only on the knowledge of the covariance matrix, the best representation of a multivariate distribution is the Gaussian distribution, in the sense that it maximizes entropy, see e.g. Cover \& Thomas (1991) for details. Therefore, conditioned on the sole knowledge of the covariance matrix $\Sigma_{\mathscr{Y}}$, the best approximated parametric representation of the multivariate distribution of $\mathscr{Y}$ is given by:

$$
f_{\mathscr{Y}}(\mathbf{y})=\frac{1}{(2 \pi)^{m / 2} \sqrt{\operatorname{det}\left(\Sigma_{\mathscr{Y}}\right)}} \exp \left(-\frac{1}{2} \mathbf{y}^{\top} \Sigma_{\mathscr{Y}}^{-1} \mathbf{y}\right)
$$

Unlike a mean-variance based normal approximation of a multivariate density, the proposed approximation heuristic preserves the fat-tailed nature of the original vector of risk factors $\mathscr{X}$. We use this method in the context of the VaR computation as follows.

If $F_{i}\left(x_{j}^{(i)}\right)$ are not normal or if $\mathscr{X}_{i}$ are not jointly normal, the VaR computation cannot be reduced to a computation of a one dimensional integral as outlined in the previous section, even after Delta-Gamma approximation of the nonlinearity. To remedy this, we carry out a nonlinear transformation to normal distributions, as proposed in Sornette et al. (2000): let $\phi$ be the standard normal pdf and $\Phi$ be the standard normal cdf. Let $f(u)$ be a density, $F(u)$ be its corresponding cdf and define the function

$$
\Phi(w)=F(u) \quad \text { i.e. } \quad \frac{1}{\sqrt{2 \pi}} \int_{0}^{w} e^{-\frac{z^{2}}{2}} d z=\int_{0}^{u} f(z) d z
$$

where the random variable $\mathscr{W} \sim \mathscr{N}(0,1)$ by construction. It is therefore possible to map each value $x_{j}$ into a new variable $y_{j}$ :

$$
y_{j}=\psi\left(x_{j}\right)=\Phi^{-1}\left(F\left(x_{j}\right)\right)=\sqrt{2} \operatorname{erf}^{-1}\left(2 F\left(x_{j}\right)-1\right)
$$

where the error function operator, erf, is defined as:

$$
\operatorname{erf}(x)=\frac{2}{\sqrt{\pi}} \int_{0}^{x} e^{-t^{2}} d t
$$

Referring back to notation introduced previously in this section since historic data is available (i.e. $x_{j}^{(i)}$, with $i=1,2, \ldots, m$ and $j=1,2, \ldots, n$ where $m$ is the number of factors and $n$ is the number of data available for each factor), it is possible to compute $y_{j}^{(i)}$ for all $i$ and $j$. The covariance matrix of the vector variable $\mathscr{Y}$ is defined as $\Sigma_{\mathscr{Y}}=\mathbb{E}\left(\mathbf{y y}^{T}\right)$, where each element $\left[\Sigma_{\mathscr{Y}}\right]_{a b}$ is obtained by sample average approximation:

$$
\left[\Sigma_{\mathscr{Y}}\right]_{a b}=\frac{1}{n} \sum_{l=1}^{n} y_{l}^{(a)} y_{l}^{(b)} .
$$

We need to find $\delta$ and $\Gamma$ in terms of the transformed variables, which will require finding the derivative $\frac{d x_{i}}{d y_{i}}$. Having $\Phi\left(\mathscr{Y}_{i}\right)=F_{i}\left(\mathscr{X}_{i}\right)$, we can write $\mathscr{X}_{i}=F_{i}^{-1}\left(\Phi\left(\mathscr{Y}_{i}\right)\right)$, where $\mathscr{Y}_{i}$ is a standard normal random variable and $\mathscr{X}_{i}$ is a random variable with cdf $F_{i}$. Since $\Phi$ and $F_{i}$ are both continuous with densities $\phi$ and $f_{i}$, respectively, it follows that the functional $\mathscr{X}_{i}=F_{i}^{-1}\left(\Phi\left(\mathscr{Y}_{i}\right)\right)$ is continuously differentiable such that:

$$
\frac{d x_{i}}{d y_{i}}=\frac{\phi\left(y_{i}\right)}{f_{i}\left(F_{i}^{-1}\left(\Phi\left(y_{i}\right)\right)\right)} \text {. }
$$


Now, remembering that $\Phi\left(\mathscr{Y}_{i}\right)=F_{i}\left(\mathscr{X}_{i}\right)$, equation $\underline{3.1}$ can be simplified as:

$$
\frac{d x_{i}}{d y_{i}}=\frac{\phi\left(y_{i}\right)}{f_{i}\left(x_{i}\right)} .
$$

Nonparametric estimation allows to achieve maximum flexibility in obtaining quantities $F_{i}\left(x_{j}^{(i)}\right)$ and $f_{i}\left(x_{j}^{(i)}\right)$; for a detailed treatment of non-parametric estimation of statistical distributions, see Bowman \& Azzalini (1997) and Fan \& Gijbels (1996).

\section{Delta-Gamma-Q for assets with non-normal returns}

Here, we bring together the material in the last two sections to propose a procedure for risk computation for a nonlinear portfolio with possibly non-Gaussian risk factors. Given a series of changes in risk factors (e.g. asset prices) $\Delta \mathscr{S}_{i}$, we start by computing their probability conserving transformation $\Delta \mathscr{Y}_{i}$ :

$$
\Delta \mathscr{Y}_{i}=\Phi^{-1}\left(F_{i}\left(\Delta \mathscr{S}_{i}\right)\right), \quad i=1,2, \ldots, m .
$$

Each $\Delta \mathscr{Y}_{i} \sim \mathscr{N}(0,1)$, and the vector $\Delta \mathscr{Y} \sim \mathscr{N}\left(0, \Sigma_{\mathscr{Y}}\right)$, with $\operatorname{diag}\left(\Sigma_{\mathscr{Y}}\right)=(1,1, \ldots, 1)$. We emphasize the fact that the matrix $\Sigma_{\mathscr{Y}}$ is generally not an identity matrix and depends on sample correlations between the elements of $\Delta \mathscr{Y}$. Since $\Delta \mathscr{Y} i$ have been represented jointly normal, VaR and CVaR computations can be estimated using an adapted version of 2.1):

$$
\Delta \Pi(\mathscr{S}, t) \approx \Theta_{\mathscr{Y}} \Delta t+\delta_{\mathscr{Y}}^{T} \Delta \mathscr{Y}+\frac{1}{2} \Delta \mathscr{Y}^{T} \Gamma_{\mathscr{Y}} \Delta \mathscr{Y},
$$

with $\Delta \mathscr{Y}$ derived from $\Delta \mathscr{X}$ using (4.1). Equation (4.2) can be converted to an one-dimensional integral only provided we can find sensitivities $\delta_{\mathscr{Y}}$ and $\Gamma_{\mathscr{Y}}$. Now, note that:

$$
\begin{gathered}
\Theta=\Theta_{\mathscr{Y}}, \\
{\left[\delta_{\mathscr{Y}}\right]_{i}=\frac{\partial \Pi}{\partial \mathscr{Y}_{i}}=\frac{\partial \Pi}{\partial \mathscr{S}_{i}} \frac{d \mathscr{S}_{i}}{d \mathscr{Y}_{i}}=[\delta]_{i} \frac{d \mathscr{S}_{i}}{d \mathscr{Y}_{i}}, \quad \text { and }} \\
{\left[\Gamma_{\mathscr{Y}}\right]_{i j}=\frac{\partial}{\partial \mathscr{Y}_{j}}\left(\frac{\partial \Pi}{\partial \mathscr{Y}_{i}}\right)=\frac{\partial}{\partial \mathscr{S}_{j}}\left(\frac{\partial \Pi}{\partial \mathscr{S}_{i}} \frac{d \mathscr{S}_{i}}{d \mathscr{Y}_{i}}\right) \frac{d \mathscr{S}_{i}}{d \mathscr{Y}_{j}}=[\Gamma]_{i j}\left(\frac{d \mathscr{S}_{i}}{d \mathscr{Y}_{i}}\right)\left(\frac{d \mathscr{S}_{i}}{d \mathscr{Y}_{j}}\right),}
\end{gathered}
$$

where $\delta$ and $\Gamma$ are sensitivities under normal conditions, while the derivatives $d \mathscr{S}_{k} / d \mathscr{Y}_{k}$ are computed using formula (3.1). Equation (4.2) has got the same structure as of equation 2.17. Hence we can apply the characteristic function inversion using the fast Fourier transform to find approximate VaR and CVaR of the portfolio using Delta-Gamma approximation. We will call this new method as Delta-Gamma-Q method for assets with non-normal returns. We summarize the steps for computation of VaR and CVaR using Delta-Gamma-Q method below:

- Find option sensitivities $\delta, \Gamma$ and $\Theta$ assuming normality for risk-factors;

- Apply nonparametric estimation to the distribution of the relevant risk-factors $\mathscr{S}$;

- Map $\Delta \mathscr{S}$ into $\Delta \mathscr{Y}$ through probability conserving transformation;

- Determine the covariance matrix $\Sigma_{\mathscr{Y}}$; 
- Find for each risk-factor the coefficient that expresses its non-normality by applying equation (3.1) and by averaging values;

- Evaluate the coefficients $\delta_{\mathscr{Y}}$ and $\Gamma_{\mathscr{Y}}$ using equations (4.4) and (4.5) respectively;

- Calculate characteristic function coefficients $a_{\mathscr{Y}}=-\Theta_{\mathscr{Y}} \Delta t, \mathbf{b}_{\mathscr{Y}}=-C_{\mathscr{Y}}^{T} \delta_{\mathscr{Y}}$ and $\Lambda_{\mathscr{Y}}=-\frac{1}{2} C_{\mathscr{Y}}^{T} \Gamma_{\mathscr{Y}} C_{\mathscr{Y}}$, where $C_{\mathscr{Y}}$ is a square root of $\Sigma_{\mathscr{Y}}$ as described in Section 2

- Compute VaR and CVaR, either by evaluating the integral in equation (2.5) directly with appropriate limits or by using FFT.

To re-emphasize the point of this exercise, we are trying to re-gain the simplicity of computing the quantiles of a quadratic form for a Gaussian distribution, while still preserving the tail information in the marginal risk factor distributions. Note, in particular, that $\Sigma_{\mathscr{Y}}$ is not an approximation to the covariance matrix of $\Delta \mathscr{S}$ as this is not a mean-variance based approximation. Approximating a distribution by a normal distribution via probability preserving transformation can lead to far better quantile estimates than a mean-variance based approximation, as the experiments in the subsequent sections show.

Note that our VaR evaluation is static, i.e. we are computing VaR over a single time-step. As such, we are using non-parametric distribution to model the risk factors and will then map them into normally distributed risk factors. The underlying stochastic process which generates the said non-parametric distribution is of no direct relevance in our current framework. Linking the evolution through time of the risk factors (e.g. as a Lévy process) to the evolution of quantiles of their multivariate nonlinear function (such as the portfolio net worth) is an interesting topic which is outside the scope of this paper and is a topic of current research.

We now demonstrate the method using a simulated portfolio first in the next section, followed by a backtesting experiment with real data in section 6

\section{Simulation experiments}

We first evaluate the Delta-Gamma-Q model for assets with non-normal returns considering a simple hypothetical portfolio $\pi$, in order to gain some insight in the performance of the proposed heuristic as compared to the performance of standard methods such as the Delta-Gamma Monte Carlo (also called partial Monte Carlo). The hypothetical portfolio is made up of one share each of $m$ correlated fattailed stocks $\mathscr{S}_{1}, \mathscr{S}_{2}, \ldots, \mathscr{S}_{m}$ and $m$ European call options $\mathscr{C}_{1}, \mathscr{C}_{2}, \ldots, \mathscr{C}_{m}$ having $\mathscr{S}_{1}, \mathscr{S}_{2}, \ldots, \mathscr{S}_{m}$ as underlying assets, respectively. Therefore the portfolio value is $\Pi=\sum_{i=1}^{m}\left(\mathscr{S}_{i}+\mathscr{C}_{i}\right)$. Each European call option has payoff (or value of the call option at expiry) given by:

$$
\max \left(\mathscr{S}_{i}(T)-K_{i}, 0\right) \quad i=1,2, \ldots, m .
$$

$\mathscr{S}_{i}(T)$ is the price of the $i$-th underlying stock at time $T$ (maturity), and $K_{i}$ is the $i$-th option strike price. Stocks $\mathscr{S}_{i}$ have been simulated using:

$$
\mathscr{S}_{i}(t)=\mathscr{S}_{i}(0) e^{\left(\mu_{i}-\sigma_{i}^{2} / 2\right) t+\sqrt{t} v_{i}} \quad i=1,2, \ldots, m,
$$

where $v=\left[v_{1}, v_{2}, \ldots, v_{m}\right]^{T}$ is obtained by first generating $w \sim\left[w_{1}, w_{2}, \ldots, w_{m}\right]^{T}$, then given the correlation matrix $H$, we set $v=C^{T} w$, where $C$ is the Cholesky factor decomposition of $H$. $w$ is chosen to have a fat-tailed distribution, the exact choice of which is discussed later in this section. The computation of each option value has been obtained using Black-Scholes formulae:

$$
\mathscr{C}(\mathscr{S}, K, \sigma, r, T-t)=\mathscr{S} \Phi\left(d_{1}\right)-K \Phi\left(d_{2}\right) e^{-r(T-t)},
$$


where

$$
d_{1}=\frac{\ln (\mathscr{S} / K)+\left(r+\frac{1}{2} \sigma^{2}\right)(T-t)}{\sigma \sqrt{T-t}}, \quad d_{2}=\frac{\ln (\mathscr{S} / K)+\left(r-\frac{1}{2} \sigma^{2}\right)(T-t)}{\sigma \sqrt{T-t}},
$$

where $T$ represents the maturity, $T-t$ is the time to maturity and $r$ is the interest rate and

$$
\Phi(x)=\frac{1}{\sqrt{2 \pi}} \int_{-\infty}^{x} e^{-\frac{1}{2} y^{2}} d y .
$$

Note that these formulae will not be valid unless $w$ above is normally distributed, i.e. unless the underlying continuous time process is geometric Brownian motion. However, option prices and sensitivities are often computed under assumption that Black-Scholes formula holds true, irrespective of evidence to the contrary, see e.g. Shao et al. (2006). Sensitivities of interest for a European call option $\mathscr{C}$ such as Delta, Gamma and Theta are given by:

$$
\begin{gathered}
\delta_{\mathscr{C}}=\frac{\partial \mathscr{C}}{\partial \mathscr{S}}=\Phi\left(d_{1}\right), \Gamma_{\mathscr{C}}=\frac{\partial^{2} \mathscr{C}}{\partial \mathscr{S}^{2}}=\frac{\phi\left(d_{1}\right)}{\mathscr{S} \sigma \sqrt{T-t}}, \\
\Theta_{\mathscr{C}}=\frac{\partial \mathscr{C}}{\partial t}=-\left[\frac{\mathscr{S} \sigma \phi\left(d_{1}\right)}{2 \sqrt{T-t}}+r K \Phi\left(d_{2}\right) e^{-r(T-t)}\right],
\end{gathered}
$$

where $\phi(x)=\frac{1}{\sqrt{2 \pi}} e^{-x^{2} / 2}$. In keeping with the standard market practice, we will use formulae (5.1)-(5.3) for prices and sensitivities in our computation. However, we assume that the real stock price dynamics are driven by fat tailed distributions rather than normal ones. The actual distributions of $w_{i}$ and the parameters used are described later in this section. While using the formulae above is mathematically inconsistent with having non-Gaussian risk factors, note that it is standard market practice to use BlackScholes formulae despite its lack of mathematical justification. As an example, Black-Scholes pricing formula is commonly used to construct an implied volatility surface for options on the same underlying asset with various strikes and maturities. This surface would be reduced to a single point if the assumptions behind the formula were to be true. Further, implied volatilities are often used for the measurement of risk using partial Monte Carlo for option portfolios. Our choice of using these expressions for $\delta_{\mathscr{C}}, \Gamma_{\mathscr{C}}$ is thus in line with the reality of the market and it also allows us to make a 'like-for-like' comparison between various partial Monte Carlo methods, as we will see next.

Numerical experiments involved comparisons of five different methods:

- Empirical method is a Monte Carlo assessment of the chosen assets. Possible $h$ day ahead portfolio values are obtained by simulating $M$ trajectories for the $m$ stocks and adding their corresponding European call values, computed through (5.2). The simulation is performed with distribution of risk factors specified later in this section. The loss and profit distribution is achieved subtracting the stocks' initial values and the fair price of the call options from the simulated portfolio values;

- Delta-Gamma-dP method, also referred to as partial Monte Carlo earlier, is a computation according to formula (2.1). This involves simulating $M$ trials for each $\mathscr{S}_{i}$, where $\Delta \mathscr{S}_{i}$ are obtained deducting $\mathscr{S}_{i}(0)$ from the corresponding stock price $\mathscr{S}_{i}$ while $\delta$ and $\Gamma$ are the ones provided in section 2 ,

- Delta-Gamma-Q method involves nonparametric estimation of pdf and cdf of $m$ risk factors. Nonparametric estimation is able to capture the features of risk factors such as skewness and 
fat-tailedness. The probability conserving transformation (4.1) on each $\Delta \mathscr{S}_{i}$ provides cdf and pdf values to apply formula $(4.2)$. Coefficients $\left[\delta_{\mathscr{Y}}\right]_{i}$ in (4.4) are computed approximately as $\left[\boldsymbol{\delta}_{\mathscr{Y}}\right]_{i} \approx$ $\mathbb{E}\left[d \mathscr{S}_{i} / d \mathscr{Y}_{i}\right][\delta]_{i}$, while elements $\left[\Gamma_{\mathscr{Y}}\right]_{i j}$ are computed approximately as

$$
\left[\Gamma_{\mathscr{Y}}\right]_{i j} \approx \mathbb{E}\left[d \mathscr{S}_{i} / d \mathscr{Y}_{i}\right] \mathbb{E}\left[d \mathscr{S}_{j} / d \mathscr{Y}_{j}\right][\Gamma]_{i j}
$$

where coefficients $d \mathscr{S}_{k} / d \mathscr{Y}_{k}$ are calculated using formula (3.2). The approximation $\mathbb{E}\left[d \mathscr{S}_{i} / d \mathscr{Y}_{i}\right]$ is introduced to decrease the computational burden of the algorithm and, simultaneously, to achieve VaR values close to those obtained through full Monte Carlo simulation. Being $\Delta \mathscr{Y}_{i} \sim$ $\mathscr{N}(0,1)$ we are in a framework similar to the one described by 2.2$]$, it is therefore possible to find coefficients $a_{\mathscr{Y}}, b_{\mathscr{Y}}$ and $\lambda_{\mathscr{Y}}$ 's. Using these coefficients one can invert the related characteristic function via FFT;

- Delta-Gamma-dP Normal method applies formula (2.1), i.e. assuming $\mathscr{S}_{i}$ and $\mathscr{C}_{i}$ to be jointly normally distributed and ignoring the functional dependence between $\mathscr{S}_{i}$ and $\mathscr{C}_{i}$;

- Delta-Gamma-Q Normal method applies formula (2.4), i.e. uses a quadratic approximation assuming normal risk factors as in Delta-Gamma-dP above, but computes VaR using an inversion integral.

The last two models use normal factors with mean and variance that match sampling the mean and the variance of changes in the respective risk factor. This methodology allows a fair comparison between the first batch of three models considered and the last two. Making this assumption can be interpreted as follows: provided that a portfolio evolves as described by the empirical model, what would be the VaR and CVaR that one would compute by employing the Delta-Gamma-Q model or a Delta-Gamma-Normal model?

Probability distributions of risk factors for empirical and Delta-Gamma-dP have to be assessed. This can be done using either parametric (making use of a preselected model fitted on the already available dataset) or nonparametric estimation. Once the probability distribution is estimated, Monte Carlo simulation can be used to find $\mathrm{VaR}$ and $\mathrm{CVaR}$ for empirical as well as Delta-Gamma-dP methods. For Delta-Gamma-dP normal and Delta-Gamma-Q-normal, VaR and CVaR are obtained by using formulae (1.1) and 1.2 for normal distribution and given confidence level.

Once VaR is computed using different methods, we wish to compare their accuracy. Confidence intervals and/or standard error estimates are usually used to perform this task. The author in Pritsker (1996) stated "This is typically not done for Delta and Delta-Gamma based estimates of VaR since there is no natural method for computing a standard error or constructing a confidence interval." about this issue. One can use the empirical distribution from a Monte Carlo simulation to obtain confidence intervals for VaR estimates (95\% confidence are typically calculated, but this can be easily generalized). The nonparametric confidence intervals, based on finite sample theory, are easy to compute and are valid for any continuous distribution of the random variable $\mathscr{L}$. Varying the sample size, $M$, of the Monte Carlo simulation changes the width of the confidence interval, according to the accuracy of VaR needed. Table 1 displays the index of ordered statistics to build $95 \%$ confidence intervals for $95 \%$ and $99 \%$ VaR for a different number of draws. Confidence intervals for CVaR can also be obtained using formula (1.2) and table 1 but are not reported here. 


\begin{tabular}{c|cc|cc}
\multicolumn{4}{c}{ Table 1. 95\% confidence intervals for Monte Carlo 95\% and 99\% VaR. } \\
\hline \multirow{2}{*}{ Number of Draws } & \multicolumn{2}{|c}{$95 \%$ VaR } & \multicolumn{3}{c}{$99 \%$ VaR } \\
\cline { 2 - 5 } & Lower Bound & Upper Bound & Lower Bound & Upper Bound \\
\hline 500 & 15 & 35 & 1 & 10 \\
1,000 & 37 & 64 & 4 & 17 \\
10,000 & 457 & 544 & 81 & 120 \\
50,000 & 2,404 & 2,597 & 456 & 545 \\
100,000 & 4,865 & 5,136 & 938 & 1,063 \\
\hline
\end{tabular}

Our experiment considered a portfolio made up of fifty stocks and fifty European call options. Stock prices were generated using $w_{i}$ distributed as Student's $t$ with different degrees of freedom $v_{i}$, ranging from 4 to 10. The values chosen for the simulation include: the time horizon is $h=1$ day, the number of simulations required is $M=10^{4}$, the interest rate $r=0.05$, the maturities $T_{i}=1$ year, for $i=1,2, \ldots, 50$. The correlation matrices $H$ are randomly generated. Strike prices $K_{i}$ and initial values $\mathscr{S}_{i}(0)$ were chosen such that some of the options were in the money, some were at the money and some were out of the money. The parameters used in our simulation are in the range reported in table 2. The full table of values is omitted for brevity.

\begin{tabular}{ccc}
\multicolumn{3}{c}{ Table 2. Parameters range. } \\
\hline Value & Min & Max \\
\hline$K_{i}$ & 2 & 99 \\
$\mu_{i}$ & -4.3 & 4.1 \\
$\mathscr{S}_{i}(0)$ & 12 & 123 \\
$v_{i}$ & 4 & 10 \\
\hline
\end{tabular}

The proposed method provides a new covariance matrix which better reflects non-normality in risk factor distributions. Computations of $95 \%$ and $99 \%$ VaR and CVaR have been obtained keeping constant all the values except from the correlation matrix $H$ which is allowed to vary and reported in tables 34

\begin{tabular}{lcccc} 
Table 3 . Values of $95 \%$ and $99 \%$ VaR for the four experiments reported, with confidence intervals in brackets. \\
\cline { 2 - 5 } Experiment & 1 & 2 & 3 & 4 \\
\hline $95 \%$ VaR & 90.4 & 72.6 & 82.1 & 160.1 \\
Empirical & $(87.9,93.3)$ & $(71.1,74.7)$ & $(79.4,84.5)$ & $(151.8,167.7)$ \\
& 92.3 & 74.1 & 82.5 & 162.6 \\
$\Delta-\Gamma$-dP & $(89.4,94.7)$ & $(71.7,76.3)$ & $(79.2,85.4)$ & $(153.2,168.4)$ \\
& 91.0 & 71.9 & 81.9 & 164.1 \\
$\Delta-\Gamma$-Q & 84.6 & 90.2 & 86.1 & 102.5 \\
$\Delta-\Gamma$-dP Normal & $(81.6,87.5)$ & $(87.4,92.8)$ & $(83.8,89.9)$ & $(96.4,107.8)$ \\
& 84.0 & 91.0 & 85.4 & 104.4 \\
$\Delta-\Gamma$-Q Normal & \multicolumn{5}{c}{} \\
\hline $99 \%$ VaR & 131.5 & 108.0 & 117.8 & 246.9 \\
Empirical & $(127.3,135.0)$ & $(103.8,111.6)$ & $(110.8,124.3)$ & $(237.2,254.5)$ \\
& 136.0 & 109.1 & 119.8 & 244.9 \\
$\Delta-\Gamma$-dP & $(131.9,140.9)$ & $(106.6,112.6)$ & $(115.2,124.6)$ & $(238.1,253.2)$ \\
& 135.4 & 109.4 & 118.2 & 250.0 \\
$\Delta-\Gamma$-Q & 127.4 & 135.6 & 126.8 & 152.0 \\
$\Delta-\Gamma$-dP Normal & $(122.2,131.6)$ & $(132.2,140.8)$ & $(121.5,132.1)$ & $(146.5,157.7)$ \\
& 127.1 & 137.4 & 125.9 & 155.7 \\
$\Delta-\Gamma$-Q Normal & \multicolumn{5}{c}{}
\end{tabular}


Table 4 . Values of $95 \%$ and $99 \%$ CVaR obtained by varying the correlation matrix.

\begin{tabular}{lcccc}
\hline Experiment & 1 & 2 & 3 & 4 \\
\hline $95 \% \mathrm{CVaR}$ & & & & \\
Empirical & 115.9 & 94.2 & 103.7 & 212.8 \\
$\Delta-\Gamma$-dP & 118.4 & 95.5 & 105.5 & 212.7 \\
$\Delta-\Gamma$-Q & 115.2 & 94.9 & 105.8 & 217.7 \\
$\Delta-\Gamma$-dP Normal & 110.0 & 117.5 & 110.8 & 132.4 \\
$\Delta-\Gamma$-Q Normal & 110.8 & 119.1 & 111.2 & 134.8 \\
\hline $99 \%$ CVaR & & & & \\
Empirical & 150.9 & 127.5 & 132.9 & 292.5 \\
$\Delta-\Gamma$-dP & 155.6 & 125.3 & 141.0 & 288.5 \\
$\Delta-\Gamma$-Q & 153.4 & 127.0 & 131.5 & 285.7 \\
$\Delta-\Gamma$-dP Normal & 147.2 & 155.2 & 143.9 & 176.9 \\
$\Delta-\Gamma$-Q Normal & 145.8 & 157.9 & 142.5 & 180.4 \\
\hline & & & &
\end{tabular}

Confidence intervals for full and partial Monte Carlo methods are obtained using values in correspondence of 10,000 draws in table 1, while interval estimates are unavailable for Delta-Gamma-Q since it is a deterministic computation. The results indicate that, with varying covariance matrix $H$, while Delta-Gamma-dP and Delta-Gamma-Q provide a reasonably good approximation to the empirical value of VaR and CVaR, the two remaining models (based on normality assumption) do not provide a consistent estimation of $\mathrm{VaR}$ and $\mathrm{CVaR}$. In particular, using the empirical VaR estimations as references, we can state that:

- in the first experiment $95 \%$ and $99 \%$ VaR are underestimated of about $7 \%$ and $3 \%$, respectively and $95 \%$ and $99 \% \mathrm{CVaR}$ are underestimated of about $5 \%$ and $3 \%$, respectively;

- in the second experiment $95 \%$ and $99 \%$ VaR are overestimated of about $25 \%$ and $26 \%$, respectively and $95 \%$ and $99 \% \mathrm{CVaR}$ are overestimated of about $25 \%$ and $23 \%$, respectively;

- in the third experiment $95 \%$ and $99 \%$ VaR are overestimated of about $4 \%$ and $7 \%$, respectively and $95 \%$ and $99 \%$ VaR are both overestimated of about $7 \%$;

- in the fourth experiment $95 \%$ and $99 \%$ VaR are underestimated of about $35 \%$ and $37 \%$, respectively and $95 \%$ and $99 \% \mathrm{CVaR}$ are underestimated of about $37 \%$ and $38 \%$, respectively.

VaR and CVaR computed under the assumption of normal distributed risk factors are, according to expectations, unreliable. Results for varying parameters other than $H$ are qualitatively similar. Portfolio composition changes such as the number of stocks included, the number and/or kind of (puts or calls) options included also lead to similar qualitative conclusions. Finally, the proposed methodology is quite general and works when few risk factors are normally distributed and/or present a certain degree of skewness. This was verified in simulation experiments with few normal and centered skew- $t$ distributions (see e.g. Azzalini \& Capitanio (2003) for details about skew- $t$ distributions) with a skewness absolute value greater than one components. Results in all these cases (varying other parameters, changing portfolio composition and changing distributional assumptions) do not add any additional information and are hence omitted for brevity. We express hereafter some considerations about accuracy and computational time of the simulation for the three methods used for computation of VaR and CVaR in our article: full Monte Carlo, partial Monte Carlo and Delta-Gamma-Q method. A fair comparison 
involves that all three methods require a nonparametric estimation. Let us recall that $m$ is the number of risky factors and $M$ is the number of simulations required. The empirical method or full Monte Carlo method is generally thought to give the most accurate estimates of VaR for large sample sizes but it tends to be very time consuming, specially when the analytical solutions for some assets are not available. Furthermore, closed-form pricing formulae are not often available and options often need to be priced (and the sensitivities need to be computed) numerically, e.g. by solving a partial differential equation. In these cases, the time required will be several orders of magnitude higher, especially for Monte Carlo method. The Delta-Gamma method or partial Monte Carlo produces estimates less accurate than full Monte Carlo but is less expensive in terms of time. As reported in Mina \& Ulmer (1999), the partial Monte Carlo requires $\mathscr{O}\left(\mathrm{Mm}^{2}\right)$ operations while the full Monte Carlo requires additional time to assess all of the positions $M$ times. The Delta-Gamma-Q method demonstrates an accuracy which is comparable to the one of partial Monte Carlo and requires $\mathscr{O}\left(\mathrm{m}^{3}\right)$ operations. Therefore, comparing computational costs it emerges that partial Monte Carlo is slower than the Delta-Gamma-Q method unless $m$ is very large depending on the VaR confidence level $\alpha$ (e.g., about 1,000 for 95\% VaR and about 5,000 for 99\% VaR, as chosen in Mina \& Ulmer (1999)). Speed performances of a typical portfolio made up of $m$ stocks and $m$ European call options with $m=1,2,3,4,5,10,20,30,40,50$ for the empirical, $\Delta-\Gamma-\mathrm{dP}$ and $\Delta-\Gamma-\mathrm{Q}$ has been measured using an Intel dual core i3 clocked at $2.66 \mathrm{GHz}$, with 3 GB RAM and using MATLAB 7.9. Any computation includes estimation of $95 \%$ and $99 \%$ VaR and $\mathrm{CVaR}$. The computational times (expressed in seconds) of the empirical, $\Delta-\Gamma$-dP and $\Delta-\Gamma-\mathrm{Q}$ methods are reported in table 5

Table 5. Net computational times for the computation of VaR and CVaR for the portfolio made up of $m$ stocks and $m$ European call options, with $m=1,2,3,4,5,10,20,30,40,50$.

\begin{tabular}{cccc}
\hline$m$ & Empirical & $\Delta-\Gamma-\mathrm{dP}$ & $\Delta-\Gamma-\mathrm{Q}$ \\
\hline 1 & 0.090184 & 0.084911 & 0.025212 \\
2 & 0.137924 & 0.103209 & 0.040598 \\
3 & 0.158297 & 0.113802 & 0.043022 \\
4 & 0.207286 & 0.137386 & 0.048445 \\
5 & 0.233043 & 0.164483 & 0.051230 \\
10 & 0.621080 & 0.206162 & 0.067723 \\
20 & 0.832268 & 0.301063 & 0.163616 \\
30 & 1.018157 & 0.373982 & 0.231480 \\
40 & 1.288235 & 0.479643 & 0.288678 \\
50 & 1.466571 & 0.563397 & 0.332650 \\
\hline
\end{tabular}

The computational times in table 5 refer to computations with 10,000 simulations and do not include the time for nonparametric estimation of the risk factors. We set the full Monte Carlo method as the reference in terms of values and computational times. It can be noticed that both partial Monte Carlo and Delta-Gamma-Q are quantitatively comparable to the reference, as shown in tables 3,4. Furthermore, our method requires a lower amount of time than the other two methods considered for all the included values of $m$. As envisaged in the study of computation costs the Delta-Gamma-Q method for the computation of results is the quickest, since it exploits the computational speed of the FFT algorithm. Larger scale computational tests tend to be portfolio-specific. However, the qualitative conclusion that Delta-Gamma-Q method is comparable in its speed to Delta-Gamma-Normal method, but outperforms it in accuracy for non-normal risk factors, holds true. 


\section{Empirical tests}

Having seen the performance of our method with a simulated portfolio with up to 50 assets, we now move to demonstrating it with a real financial portfolio and comparing it with Delta-Gamma-Normal method in computing VaR and CVaR. This section is divided into five subsections. Subsection 6.1 introduces the data employed and presents some of their descriptive statistics. The portfolio analyzed is constituted of four European options on FTSE100 index (which represents our single risk factor). A representative real portfolio with a relatively small number of assets is chosen for demonstration since it is easier to visualize data and report results with a small number of assets; it is also easy to reproduce our experiments based on the information provided here, if desired. Subsection 6.2 illustrates the choice and the features of the portfolio tested, while section 6.3 includes details on the backtesting tools used. These include tests for unconditional as well as conditional coverage in predicting tail losses. Subsections 6.4 and 6.5 respectively report the results of backtesting using Delta-Gamma-Normal approach and the approach presented in this paper.

\subsection{Data}

For computing the VaR estimates and for backtesting, we use two sets of data:

1. A set of 501 daily closing prices for each of the four included European options on the underlying index FTSE100, according to the portfolio composition specified in tables 7,8

2. A set of daily closing FTSE100 values which is used for estimating the parameters related to non-normality as described in section 3

All the data has been retrieved from Datastream. The portfolio analyzed includes observations from $10 / 07 / 2009$ to $10 / 06 / 2011$. The whole set of daily portfolio variations has been split in two 250 units subsets: an in-sample subset that covers observations from 13/07/2009 to 25/06/2010, and an out-of-sample subset that covers observations from 28/06/2010 to 10/06/2011. Summary statistics on FTSE100 daily returns $R_{t}$ for the considered period are reported in table 6 .

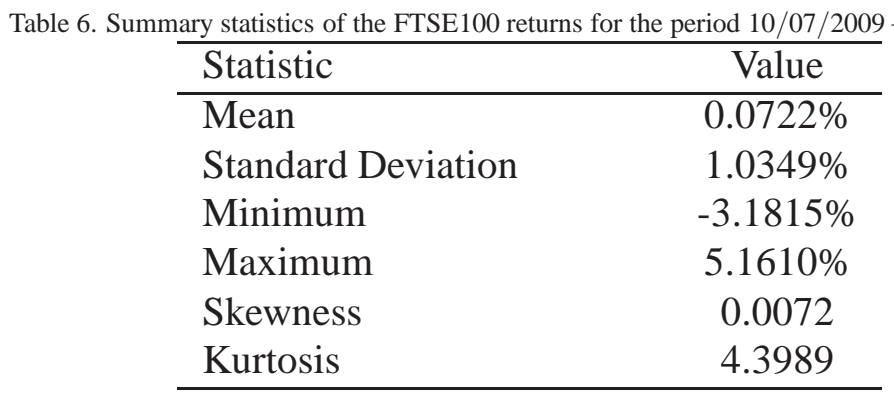

The empirical distribution of daily returns is leptokurtic (i.e., its kurtosis exceeds the value 3), that indicates fat-tailedness, and slightly skewed. The Lilliefors test, used to test the null hypothesis that data come from a normal distribution when the sample is small (see e.g. Lilliefors (1967) for details), also rejects normality at both $5 \%$ and $1 \%$ significance level for the considered series. Figure1 1 represents the returns histogram for FTSE100 for the considered period. 


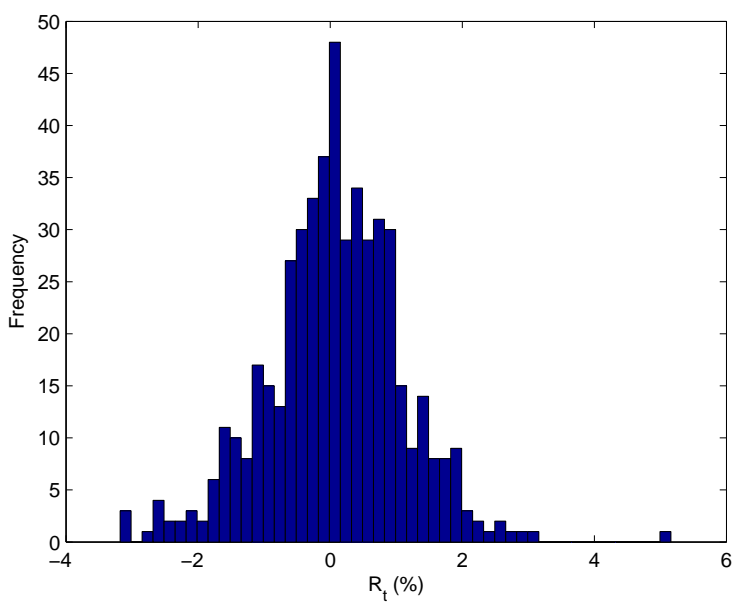

FIG. 1. FTSE100 returns histogram for the period 10/07/2009 - 10/06/2011.

\subsection{Test portfolio}

The portfolio tested includes four European options on the same underlying: the index FTSE100. Its composition is changed periodically to mirror the variations occurring in a typical, actively traded options portfolio. To be specific, the portfolio is made up of two pairs of options, each pair consisting of a call option and one put option having common strike price $K$ and maturity $T$. The daily portfolio $\pi_{t}$ is made up of $\mathscr{C}_{t}^{(1)}, \mathscr{P}_{t}^{(1)}, \mathscr{C}_{t}^{(2)}$ and $\mathscr{P}_{t}^{(2)}$, with $t=1,2, \ldots, 501$. Therefore the daily portfolio values $\Pi_{t}$ are computed as $\Pi_{t}=\mathscr{C}_{t}^{(1)}+\mathscr{P}_{t}^{(1)}+\mathscr{C}_{t}^{(2)}+\mathscr{P}_{t}^{(2)}$ and the daily changes in portfolio values are computed as $\Delta \Pi_{t-1}=\Pi_{t}-\Pi_{t-1}$ for $t=2,3, \ldots, 501$. We decided to change the portfolio composition over time, including options having different characteristics (strike prices and maturities), to highlight that the method does not depend on specific features of the portfolio. As mentioned earlier, nonlinear portfolios are rarely static over a long period of time. To generate a large enough data sample, with the same underlying risk factor for backtesting, it makes sense to use a portfolio of options which evolves over time. The whole length of time under test is split in six different length intervals. Intervals with different lengths provide again a more general framework. At every change of interval one of the two call-put pairs is dropped and is alternately replaced by another call-put pair of options, with both the call and the put having the same strike price and maturity. Table 7 displays the portfolio composition chosen, whereas figure 2 provides a graphical representation of the portfolio composition as a function of time. 


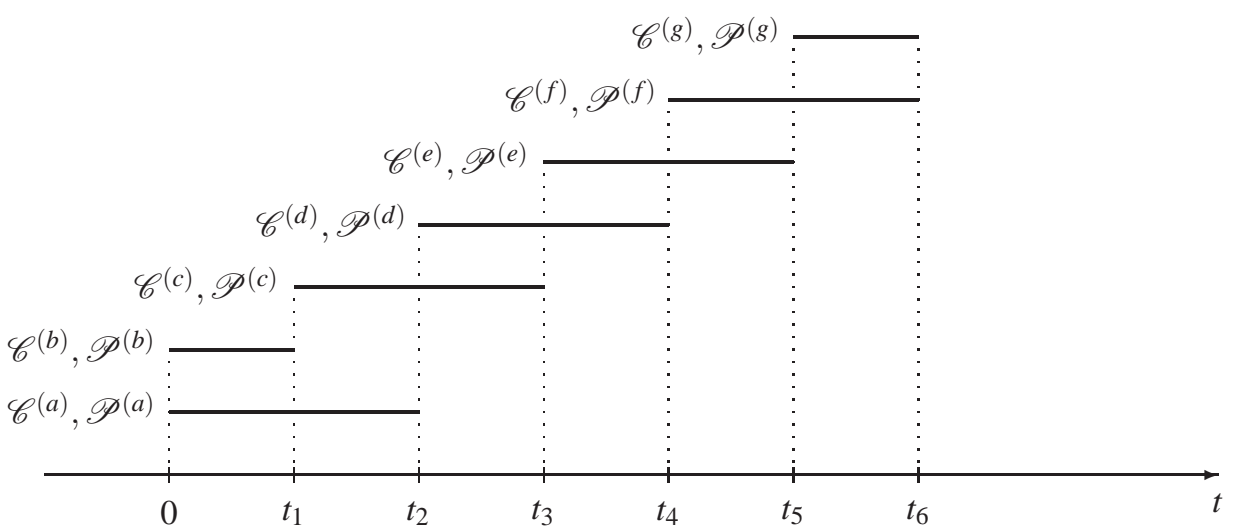

FIG. 2. Graphical representation of portfolio composition in time. Options are included in the portfolio in correspondence of the relative thick segment.

Note that most options have a life which is shorter than what one needs for a reasonably large backtesting data sample, which makes the proposed changes in portfolio over time (keeping the risk factor the same) a sensible alternative for backtesting VaR methodologies.

Table 7. Portfolio composition in the different intervals.

\begin{tabular}{cccccccc}
\hline Interval & from & to & Length & $\mathscr{C}_{t}^{(1)}$ & $\mathscr{P}_{t}^{(1)}$ & $\mathscr{C}_{t}^{(2)}$ & $\mathscr{P}_{t}^{(2)}$ \\
\hline 1 & $10 / 07 / 2009$ & $10 / 11 / 2009$ & 88 & $\mathscr{C}^{(a)}$ & $\mathscr{P}^{(a)}$ & $\mathscr{C}^{(b)}$ & $\mathscr{P}^{(b)}$ \\
\hline 2 & $11 / 11 / 2009$ & $26 / 02 / 2010$ & 78 & $\mathscr{C}^{(a)}$ & $\mathscr{P}^{(a)}$ & $\mathscr{C}^{(c)}$ & $\mathscr{P}^{(c)}$ \\
\hline 3 & $01 / 03 / 2010$ & $30 / 07 / 2010$ & 110 & $\mathscr{C}^{(d)}$ & $\mathscr{P}^{(d)}$ & $\mathscr{C}^{(c)}$ & $\mathscr{P}^{(c)}$ \\
\hline 4 & $02 / 08 / 2010$ & $30 / 11 / 2010$ & 87 & $\mathscr{C}^{(d)}$ & $\mathscr{P}^{(d)}$ & $\mathscr{C}^{(e)}$ & $\mathscr{P}^{(e)}$ \\
\hline 5 & $01 / 12 / 2010$ & $15 / 03 / 2011$ & 75 & $\mathscr{C}^{(f)}$ & $\mathscr{P}^{(f)}$ & $\mathscr{C}^{(e)}$ & $\mathscr{P}^{(e)}$ \\
\hline 6 & $16 / 03 / 2011$ & $10 / 06 / 2011$ & 63 & $\mathscr{C}^{(f)}$ & $\mathscr{P}^{(f)}$ & $\mathscr{C}^{(g)}$ & $\mathscr{P}^{(g)}$ \\
\hline
\end{tabular}

Strike prices and maturities of each pair of options $\mathscr{C}^{(l)}, \mathscr{P}^{(l)}, l \in\{a, b, c, d, e, f, g\}$ are reported in table 8

Table 8. Considered strike prices and maturities.

\begin{tabular}{lccccccc}
\hline Asset Label & $\mathrm{a}$ & $\mathrm{b}$ & $\mathrm{c}$ & $\mathrm{d}$ & $\mathrm{e}$ & $\mathrm{f}$ & $\mathrm{g}$ \\
\hline Strike Price & 3,800 & 2,000 & 3,000 & 4,800 & 3,700 & 5,400 & 2,600 \\
\hline Maturity & $18 / 06 / 10$ & $18 / 06 / 10$ & $17 / 09 / 10$ & $17 / 12 / 10$ & $18 / 03 / 11$ & $17 / 06 / 11$ & $17 / 06 / 11$ \\
\hline
\end{tabular}

\subsection{Backtesting: methodology}

Backtesting is a statistical tool to verify whether a model is adequate for its purpose. In the case of VaR models, it consists in checking that actual losses are in line with projected ones. It is crucial to check if 
predicted values of measure of risk are reliable. If that is not the case one should reassess assumptions, include a different - and valid - set of parameters or provide an improved modeling methodology. In this section we are backtesting VaR computations. Several authors recommend backtesting VaR models including Jorion (2007), Kupied (1995) and Christoffersen (2003). The most common method to test a VaR model has been suggested in Kupiec (1995), where the author developed a 95\% confidence region for the unconditional coverage test. The unconditional coverage test is the standard tool for backtesting models and is also recommended by Basel II (see, e.g. Chen \& Gerlach (2011)), therefore we decided to employ it throughout this paper. According to this procedure, a model is correctly calibrated when the number of exceptions (i.e. portfolio losses exceeding VaR) is in line with the confidence level. If backtesting reveals too many exceptions then the risk is underestimated by the current model, hence one could reserve an insufficient required capital and suffer critical losses under extreme market movements. On the other hand too few exceptions signals an overestimated risk and that would lead to an inefficient allocation of capital. This situation is also not ideal for institutions that look for maximizing profits. Let's define $\mathscr{I}_{t}$ as:

$$
\mathscr{I}_{t}= \begin{cases}0 & \text { if } \mathscr{L}_{t} \leqslant V a R_{\alpha, t \mid t-1} \\ 1 & \text { if } \mathscr{L}_{t}>V a R_{\alpha, t \mid t-1}\end{cases}
$$

where $\mathscr{L}_{t}$ and $\operatorname{VaR} R_{\alpha, t \mid t-1}$ represent respectively the loss at time $t$ and the $\alpha$ confidence level VaR computed at time $t$ given the information at time $t-1$. Therefore the number of exceptions is given by $\mathscr{X}=\sum_{t=1}^{N} \mathscr{I}_{t}$, where $N$ is the total number of observations. Since each daily outcome could lead to an exception or not, the random variable $\mathscr{X}$ follows a binomial distribution:

$$
f_{\mathscr{X}}(x)=\left(\begin{array}{c}
N \\
x
\end{array}\right) p^{x}(1-p)^{N-x},
$$

where $p=1-\alpha$, and $\alpha$ is the level for the selected VaR. Let us consider the number of exceptions in the sample, $\tilde{x}$, and define the failure rate as $\tilde{x} / N$. Null and alternative hypothesis are in Kupiec's test Kupiec (1995)) are as follows:

$$
\left\{\begin{array}{l}
H_{0}: p=\frac{\tilde{x}}{N} \\
H_{1}: p \neq \frac{\tilde{x}}{N}
\end{array}\right.
$$

so we test whether the observed failure rate differs significantly from the given confidence level $p$. The test statistic used is:

$$
L R_{u c}=-2 \ln \left(\frac{(1-p)^{N-\tilde{x}} p^{\tilde{x}}}{\left[1-\frac{\tilde{x}}{N}\right]^{N-\tilde{x}}\left(\frac{\tilde{x}}{N}\right)^{\tilde{x}}}\right) \sim \chi_{1}^{2} .
$$

Using a 95\% confidence interval this likelihood ratio test rejects the null hypothesis if $L R_{u c}>3.841$. Table 9 displays 95\% confidence regions of non rejection for the Kupiec's test:

\begin{tabular}{cccc}
\multicolumn{4}{c}{ Table 9. Non rejection regions for Kupiec's test. } \\
\hline$\alpha$ & $\mathrm{N}=250$ & $\mathrm{~N}=500$ & $\mathrm{~N}=1,000$ \\
\hline $95 \%$ & $7 \leqslant \tilde{x} \leqslant 19$ & $17 \leqslant \tilde{x} \leqslant 35$ & $38 \leqslant \tilde{x} \leqslant 64$ \\
\hline $99 \%$ & $1 \leqslant \tilde{x} \leqslant 6$ & $2 \leqslant \tilde{x} \leqslant 9$ & $5 \leqslant \tilde{x} \leqslant 16$ \\
\hline
\end{tabular}

The unconditional coverage test, on its own, focusses on the number of exceptions, but it does not consider whether they are clustered. Since large losses bunched in a small amount of time are more likely to cause disastrous events than single exceptions showing up occasionally (see Campbell (2005) for further details), it is crucial that the VaR model satisfies the independence property. The independence 
test, developed in Christoffersen (2003), is capable of rejecting a VaR with clustered exceptions. Let us define the indicator variable:

$$
\mathscr{J}_{t}=\left\{\begin{array}{cc}
1 & \text { if an exception occurs } \\
0 & \text { otherwise }
\end{array}\right.
$$

and then define the transition probabilities $\pi_{i j}=P\left(\mathscr{J}_{t}=i\right.$ and $\left.\mathscr{J}_{t+1}=j\right)$. As an example, $\pi_{01}$ provides the probability of having an exception tomorrow given that today there were no exception. The firstorder Markov sequence with transition probability matrix is given by:

$$
\Pi=\left[\begin{array}{ll}
\pi_{00} & \pi_{01} \\
\pi_{10} & \pi_{11}
\end{array}\right]=\left[\begin{array}{ll}
1-\pi_{01} & \pi_{01} \\
1-\pi_{11} & \pi_{11}
\end{array}\right] .
$$

If the exceptions sequence is independent over time then the probability of an exception tomorrow does not depend on today's outcome, i.e. $\pi_{01}=\pi_{11}=\pi$. In this case null and alternative hypothesis are:

$$
\left\{\begin{array}{l}
H_{0}: \pi_{01}=\pi_{11} \\
H_{1}: \pi_{01} \neq \pi_{11}
\end{array}\right.
$$

To test it we use the following likelihood ratio test:

$$
L R_{\text {ind }}=-2 \ln \left(\frac{(1-\hat{\pi})^{N_{00}+N_{10}} \hat{\pi}^{N_{01}+N_{11}}}{\left(1-\hat{\pi}_{01}\right)^{N_{00}} \hat{\pi}_{01}^{N_{01}}\left(1-\hat{\pi}_{11}\right)^{N_{10}} \hat{\pi}_{11}^{N_{11}}}\right) \sim \chi_{1}^{2},
$$

where $\hat{\pi}=\frac{N_{01}+N_{11}}{N_{00}+N_{01}+N_{10}+N_{11}}, \hat{\pi}_{01}=\frac{N_{01}}{N_{00}+N_{01}}$ and $\hat{\pi}_{11}=\frac{N_{11}}{N_{10}+N_{11}} . N_{i j}$ represents the number of days when state $j$ follows state $i$, and $i, j$ can only assume values 0 and 1 . Since we are interested in understanding whether simultaneously the number of exceptions is correct and VaR exceptions are independent, we can test jointly this two features using the conditional coverage test:

$$
L R_{c c}=L R_{u c}+L R_{\text {ind }} \sim \chi_{2}^{2} .
$$

Using a 95\% confidence interval this likelihood ratio test rejects the null hypothesis if $L R_{c c}>5.991$. Hence, the 95\% level critical values for $L R_{u c}, L R_{\text {ind }}$ and $L R_{c c}$ are 3.841,3.841 and 5.991 respectively. Computation of statistics $L R_{u c}$ and $L R_{\text {ind }}$ as respectively specified in (6.1) and (6.2) provides the tool to accept or reject the model specification. In the following sections 6.4 and 6.5 the daily estimates of $95 \%$ and $99 \%$ VaR are calculated using Delta-Gamma-Normal method and Delta-Gamma-Q method for an asset with non-normal returns, respectively, and then are compared to actual losses. The series of actual daily portfolio losses are computed as $\mathscr{L}_{t}=-\Delta \Pi_{t}$, for $t=1,2, \ldots, 500$. As mentioned earlier in section 5 , we focus on the point estimates as interval estimates are unavailable for Delta-Gamma-Q. However, the point estimates for both in-sample and out-of-sample VaR are computed for $95 \%$ as well as $99 \%$ confidence level, and are validated using unconditional as well as conditional coverage tests. The consistency of qualitative aspects of our conclusions across all these tests as well as across simulation experiments in the previous section gives us some confidence in the validity of our point estimate-based results. The results of using our method are detailed in section 6.5 , although we start with the description of results using the traditional Delta-Gamma-Normal heuristic next.

\subsection{Applying the Delta-Gamma-Normal method: results}

The first experiment consists in computing $95 \%$ and $99 \%$ VaR of the considered portfolio using DeltaGamma-Normal model and assessing its reliability through unconditional and conditional tests. The 
descriptive statistics of $\Delta \mathscr{S}$ are gathered in table 10

Table 10. Descriptive statistics of the $\Delta \mathscr{S}$.

\begin{tabular}{lcc}
\hline Statistic & In-sample & Out-of-sample \\
\hline Mean & 3.6772 & 2.8773 \\
Standard Deviation & 57.6257 & 53.3055 \\
Minimum & -170.8800 & -157.4600 \\
Maximum & 264.4000 & 141.4700 \\
Skewness & -0.1112 & -0.0386 \\
Kurtosis & 4.6705 & 3.3710 \\
\hline
\end{tabular}

A glance at the statistics reported in table 10 suggests that $\Delta \mathscr{S}$ has a different type of distribution in the two different subsets: the in-sample subset appears not normally distributed and its Lilliefors test rejects at levels $5 \%$ and $1 \%$ that $\Delta \mathscr{S}$ comes from a distribution in the normal family, while for the out-of-sample subset, the Lilliefors test does not reject at levels $5 \%$ and $1 \%$ that $\Delta \mathscr{S}$ comes from a distribution in the normal family.

Sensitivities $\Theta_{t}^{(i)}, \delta_{t}^{(i)}$ and $\Gamma_{t}^{(i)}$, with $i \in\left\{\mathscr{C}^{(1)}, \mathscr{P}^{(1)}, \mathscr{C}^{(2)}, \mathscr{P}^{(2)}\right\}$, according to the portfolio composition reported in table 7 are computed for both the in-sample data and out-of-sample data using steps (1) and (2) from the procedure described in subsection 6.5. $\Theta_{t}, \delta_{t}$ and $\Gamma_{t}$ are derived summing up the correspondent sensitivities of the options included at time $t$. The Delta-Gamma-Normal approximation becomes therefore:

$$
\Delta \Pi_{t}=\Theta_{t} \Delta t+\delta_{t} \Delta \mathscr{S}+\frac{1}{2} \Gamma_{t} \Delta \mathscr{S}^{2}
$$

Assuming that $\Delta \mathscr{S} \sim \mathscr{N}\left(0, \sigma^{2}\right)$, it is possible to use the transformation $\Delta \mathscr{S}=\sigma \mathscr{Z}$, where $\mathscr{Z} \sim$ $\mathscr{N}(0,1)$. The equation 6.4 can be rewritten as:

$$
\Delta \Pi_{t}=\Theta_{t} \Delta t+\delta_{t} \sigma \mathscr{Z}+\frac{1}{2} \Gamma_{t} \sigma^{2} \mathscr{Z}^{2} .
$$

For each of the subsets one can utilize the standard deviation of $\Delta \mathscr{S}$ and perform the partial Monte Carlo Delta-Gamma-Normal VaR. The time horizon $h$ is set to 1 day. Conditional and unconditional tests are reported in table 11

Table 11. Summary of test results for the Delta-Gamma-Normal model.

\begin{tabular}{cccccccccc}
\hline Subset & $\alpha$ & $\tilde{x}$ & $N_{00}$ & $N_{01}$ & $N_{10}$ & $N_{11}$ & $L R_{u c}$ & $L R_{\text {ind }}$ & $L R_{c c}$ \\
\hline \multirow{2}{*}{ In-sample } & $95 \%$ & 14 & 222 & 13 & 13 & 2 & 0.1827 & 1.1758 & 1.3385 \\
& $99 \%$ & 10 & 230 & 10 & 10 & 0 & 12.9555 & 0.8336 & 13.7891 \\
\hline \multirow{2}{*}{ Out-of-sample } & $95 \%$ & 15 & 222 & 13 & 14 & 1 & 0.4961 & 0.0326 & 0.5286 \\
& $99 \%$ & 4 & 242 & 4 & 4 & 0 & 0.7691 & 0.1301 & 0.8992 \\
\hline
\end{tabular}

Statistics for the in-sample subset show that the $95 \%$ VaR estimate is acceptable being all the values $L R_{u c}, L R_{\text {ind }}$ and $L R_{c c}$ below the respective critical values. Instead, the large number of failures and 
relative statistics suggest that the estimation using the Delta-Gamma-Normal method for the $99 \%$ VaR is not appropriate. This result confirms the insight that just using the standard deviation of a risk factor, neglecting its fat-tailed behavior could lead to an acceptable VaR for relative low levels of $\alpha$ (up to $95 \%$ ), but could fail to provide suitable VaR for higher values of $\alpha$, underestimating its actual value. This might explain a fraction of what has happened during the recent financial crisis: managers, having relied on Gaussian-based models and ignoring the non-normality of risk factors, obtained VaR values that were (even catastrophically) wrong. The statistics $L R_{u c}, L R_{\text {ind }}$ and $L R_{c c}$ for the out-of-sample subset are all below the respective critical values for both the $\alpha$ levels considered. This is not surprising since the out-of-sample dataset is close to being normal; also see table 10.

\subsection{Applying the Delta-Gamma-Q method: results}

The second experiment involves two phases:

- Calibrate the parameters of the Delta-Gamma-Q model for a non-normal factor using in-sample data;

- Assessing the model validity through conditional and unconditional tests using out-of-sample data.

Daily 1-day horizon $V_{a} R_{t \mid t-1}$ estimates are obtained using the appropriate option prices occurred on day $t-1$. The first 250-unit subset of losses, covering the period from $13 / 07 / 2009$ to $25 / 06 / 2010$, is used for parameter calibration while the second subset of losses, covering the period from 28/06/2010 to $10 / 06 / 2011$ is used for model validation using the parameter $D=\mathbb{E}[d \mathscr{S} / d \mathscr{Y}]$ estimated in the insample subset. The following part describes how the estimates $V a R_{t \mid t-1}$ are computed. The payoff an European call option is given by (5.1), while the payoff for an European put option is given by:

$$
\max (K-\mathscr{S}(T), 0)
$$

The calibration step itself requires the following steps for each time interval considered:

1. finding the implied risk-free rates which are needed in finding $\delta$ and $\Gamma$;

2. determining sensitivities $\delta, \Gamma$ and $\Theta$;

3. working out coefficients that capture the index FTSE100 non-normality;

4. calculating coefficients $\delta_{\mathscr{Y}}, \Gamma_{\mathscr{Y}}$ and $\Theta_{\mathscr{Y}}$;

5. deriving coefficients $a_{\mathscr{Y}}, b_{\mathscr{Y}}$ and $\lambda_{\mathscr{Y}}$.

Given that daily implied volatilities of the call and the put option in each pair, $\sigma_{\mathscr{C}}$ and $\sigma_{\mathscr{P}}$, are also available data, one can estimate the risk-free rate for each pair of options by minimizing the quantity:

$$
\left[\left(\mathscr{C}_{i}-\mathscr{C}\left(\mathscr{S}, K, \sigma_{\mathscr{C}}, r, T-t\right)\right)^{2}+\left(\mathscr{P}_{i}-\mathscr{P}\left(\mathscr{S}, K, \sigma_{\mathscr{P}}, r, T-t\right)\right)^{2}\right], \quad i=1,2,
$$

where $r$ is the only unknown, since option values $\mathscr{C}_{i}$ and $\mathscr{P}_{i}$, index value $\mathscr{S}$, strike price $K$ and time to maturity $T-t$ are all known. The function $\mathscr{C}(\cdot)$ refers to the Black-Scholes formulae to compute European call option prices and is provided by $(5.2)$, while the function $\mathscr{P}(\cdot)$ refers to the Black-Scholes formulae to compute European put option and is given by:

$$
\mathscr{P}(\mathscr{S}, K, \sigma, r, T-t)=K e^{-r(T-t)} \Phi\left(-d_{2}\right)-\mathscr{S} \Phi\left(-d_{1}\right),
$$


where $d_{1}$ and $d_{2}$ are the ones expressed in (5.2). Functional dependence of variables on $t$ is suppressed for notational brevity. Therefore two risk-free rates $r_{1}$ and $r_{2}$ are obtained for the two call-put option pairs. This minimization was carried out in MATLAB 7.9 using an inbuilt routine fminbnd, which uses golden section search and parabolic interpolation. Sensitivities Delta, Gamma and Theta for European call options can be calculated using (5.3), while corresponding sensitivities for European put options are given by:

$$
\begin{aligned}
& \delta_{\mathscr{P}}=\frac{\partial \mathscr{P}}{\partial \mathscr{S}}=\Phi\left(d_{1}\right)-1, \Gamma_{\mathscr{P}}=\frac{\partial^{2} \mathscr{P}}{\partial \mathscr{S}^{2}}=\frac{\phi\left(d_{1}\right)}{\mathscr{S} \sigma \sqrt{T-t}} \\
& \Theta_{\mathscr{P}}=\frac{\partial \mathscr{P}}{\partial t}=-\frac{\mathscr{S} \sigma \phi\left(d_{1}\right)}{2 \sqrt{T-t}}+r K \Phi\left(-d_{2}\right) e^{-r(T-t)}
\end{aligned}
$$

Having two pairs of options in our test portfolio as described in section 6.2 we obtain two sets of sensitivities applying the appropriate risk-free rate to the corresponding pair of options.

A crucial role is played by the coefficient $D=\mathbb{E}[d \mathscr{S} / d \mathscr{Y}]$ which is able to encapsulate the possible non-normality of the risk factor $\mathscr{S}$. Its value for the computation of $\operatorname{VaR}_{t}$ would be computed using a sample average over a "window" of FTSE100 data of length $n$. Using trial and error in the in-sample subset we found that a suitable width for this risk factor window $\bar{n}$ is 150 . We used three 150 wide rolling windows for the in-sample subset: the first from 10/07/2009 to 04/02/2010, the second from $18 / 09 / 2009$ to $15 / 04 / 2010$, the third from $27 / 11 / 2009$ to $25 / 06 / 2010$. The value $D$ has been computed for each rolling window using formula (3.1) and their average value was used to verify whether the model is valid using the conditional and unconditional coverage tests for the in-sample subset. This simple moving average heuristic with overlapping windows provides a smoothing effect. For assessment of out-of-sample data, $D$ is computed using a single window of width 150 from $27 / 11 / 2009$ to 25/06/2010 (i.e., the in-sample data immediately prior to the start of out-of-sample data set). The assessment of modeling for out-of-sample data is done using a window of width 150 from 27/11/2009 to $25 / 06 / 2010$, for the computation of the value $D$ needed for the conditional and unconditional coverage tests.

Coefficients $a_{\mathscr{Y}}, b_{\mathscr{Y}}$ and $\lambda_{\mathscr{Y}}$ are calculated using formulae as reported in Section 4 Results for the in-sample Delta-Gamma-Q and for the out-of-sample Delta-Gamma-Q are shown in table 12.

Table 12. Summary of test results for the Delta-Gamma-Q modeling of real data.

\begin{tabular}{cccccccccc}
\hline Subset & $\alpha$ & $\tilde{x}$ & $N_{00}$ & $N_{01}$ & $N_{10}$ & $N_{11}$ & $L R_{u c}$ & $L R_{\text {ind }}$ & $L R_{c c}$ \\
\hline \multirow{2}{*}{ In-sample } & $95 \%$ & 14 & 223 & 13 & 13 & 1 & 0.1827 & 0.0620 & 0.2447 \\
& $99 \%$ & 5 & 240 & 5 & 5 & 0 & 1.9568 & 0.2041 & 2.1609 \\
\hline \multirow{2}{*}{ Out-of-sample } & $95 \%$ & 14 & 223 & 14 & 13 & 0 & 0.1827 & 1.5400 & 1.7226 \\
& $99 \%$ & 2 & 246 & 2 & 2 & 0 & 0.1084 & 0.0323 & 0.1407 \\
\hline
\end{tabular}

Results, computed using formulae mentioned earlier in this section, show that all tests for both $95 \%$ VaR and $99 \%$ VaR are below the respective critical values, therefore cannot be rejected. The proposed model hence seems to provide acceptable VaR estimates in both the sub-samples considered and for both the confidence intervals, in contrast with Delta-Gamma-Normal method outlined in subsection 6.4 earlier. The results using 5-day time horizon were found to be consistent with these findings and are omitted for brevity. 


\section{Conclusions and future research directions}

Computing measures of risk such as $\mathrm{VaR}$ and $\mathrm{CVaR}$ involves a trade-off between accuracy and computational complexity. The method developed in this article, Delta-Gamma-Q for assets with possibly nonnormal returns, allows us to compute VaR and CVaR through a combination of Delta-Gamma-Normal model in Glasserman (2003) and probability conserving transformation in Sornette et al. (2000). In this method, the marginal distributions of risk factors are mapped through nonlinear changes of variables onto Gaussian distributions. A new covariance matrix can therefore be computed and it redefines the dependence among transformed risk factors. Delta-Gamma coefficients obtained under normal conditions are multiplied by factors that take in account the shape of risk factors and then fast Fourier transform allows us to perform a quick computation of $\mathrm{VaR}$ and $\mathrm{CVaR}$ values. Using comprehensive numerical experiments based on both simulated as well as real data, we have demonstrated that using Delta-Gamma-Normal method for non-normal risk factors leads to misleading results while our method corrects the bias to a significant extent. The numerical results are shown to be consistent across a range of parameter values, across two different confidence levels and across two different time horizons. The method presented here highlights that using methods that do not recognize the lack of normality can lead to rather biased estimates of both VaR and CVaR, especially in nonlinear portfolios. The method presented features a good degree of flexibility since the use of nonparametric estimation can capture the distribution characteristics of risk factors to be analyzed and the computational effort is lower than partial Monte Carlo simulation.

This paper focusses only on dealing with possible skewness and fat tails of the portfolio distribution. As mentioned in section 4 it would be interesting to see if the proposed framework can be extended to prediction of VaR in a dynamic setting, perhaps including phenomena such as autoregressive behavior and clustering of volatility. Further, the current work is limited to analysis of a given (or fixed) portfolio. The use of probability conserving transformation and the subsequent transformed correlations in selecting a portfolio which optimizes risk/return characteristics also indicates a potentially interesting direction for future research.

\section{References}

ACERBI, C. \& TASCHE, D. (2001) Expected Shortfall: a natural coherent alternative to Value at Risk, Economic Notes, 31:379-388.

Albanese, C., Jackson, K. \& Wiberg, P. (2004) A new Fourier transform algorithm for Value-atRisk, Quantit. Financ., 4(3):328-338.

ALeXANDer, C. (2008) Market risk analysis. Vol. 4, Value-at-Risk models. John Wiley \& Sons, Ltd.

Artzner, P., Delbaen, F., Eber, J.M. \& Heath, D. (1999) Coherent measures of risk, Math. Financ., 9(3):203-228.

Azzalini, A. \& CAPitanio, A. (2003) Distributions generated by perturbation of symmetry with emphasis on a multivariate skew- $t$ distribution, J. Roy. Stat. Soc. B, 65(2):367-389.

BASEL CoMmitTeE (2006) Basel Committee on banking supervision international convergence of capital measurement and capital standards. http://www.bis.org/publ/bcbs128.pdf. 
Bowman, A.W. \& AzZalini, A. (1997) Applied Smoothing Techniques for Data Analysis: The Kernel Approach with S-Plus Illustrations. Oxford Science Publications.

Britten-Jones, M. \& Schaefer, S.M. (1999) Non-linear Value-at-Risk, European Finance Review, 2:161-187.

CAmpBell, S.D. (2005) A review of backtesting and backtesting procedures. Working paper in Finance and Economic Discussion series, Divisions of Research \& Statistics and Monetary Affairs, Fedaral Reserve Board, Washington D.C., USA.

Castellacci, G. \& Siclari, M.J. (2003) The practice of Delta-Gamma VaR: Implementing the quadratic portfolio model, Eur. J. Oper. Res., 150(3):529-545.

Chen, Q. \& Gerlach, R.H. (2011) The two-sided Weibull distribution and the forecasting of financial risk, OME Working Paper, 1-36.

CHRISTOFFERSEN, P.F. (2003) Elements of financial risk management. Academic Press.

Cover, T.M. \& Thomas, J.A. (1991) Elements of information theory. Wiley-Interscience, New York, NY, USA.

Duffie, D. \& Pan, J. (1997) An overview of Value at Risk, J. Deriv., 4(3):7-49.

El-Jahel, L., Perraudin, W. \& Sellin, P. (1999) Value-at-Risk for derivatives, J. Deriv., 6(3):726.

Embrechts, P., Resnich, S.I. \& SAmorodnitsky, G. (1999) Extreme value theory as a risk management tool, North American Actuarial Journal, 3(2):30-41.

FAn, J. \& GiJbels, I. (1996) Local Polynomial Modelling and Its Applications. Chapman \& Hall / CRC.

Feuerverger, A. \& Wong, A.C.M. (2000) Computation of Value-at-Risk for nonlinear portfolios, J. Risk, 3(1):37-55.

Gilli, M. \& KËLlEZI, E. (2006) An application of extreme value theory for measuring financial risk, Comput. Econ., 27(5):207-228.

Glasserman, P. (2003) Monte Carlo Methods in Financial Engineering (Stochastic Modelling and Applied Probability). Springer.

Glasserman, P., Heidelberger, P. \& Shahabuddin, P. (2000) Portfolio Value-at-Risk with heavy-tailed risk factors, Math. Financ., 12:239-269.

Hull, J.C. (2006) Options, futures, and other derivatives. Pearson Prentice Hall.

JASCHKE, S.R. (2001) The Cornish-Fisher-expansion in the context of Delta-Gamma-normal approximations, J. Risk, 4(4):1-14.

JORION, P. (2007) Value at Risk: the new benchmark for managing financial risk. McGraw-Hill.

KAmDEM, J.S. (2003) Value-at-Risk and Expected Shortfall for quadratic portfolio of securities with mixture of elliptic distributed risk factors. http://arxiv.org/abs/cs.CE/0310043. 
KuPIEC, P.H. (1995) Techniques for verifying the accuracy of risk measurement models, The J. Deriv., 3(2):73-84.

Lilliefors, H.W. (1967) On the Kolmogorov-Smirnov test for normality with mean and variance unknown, J. Am. Stat. Assoc., 62:399-402.

MinA, J. \& Ulmer, A. (1999) Delta-Gamma four ways. http://www.texnology.com/risk.pdf.

Montero, M. \& Kohatsu-Higa, A. (2003) Malliavin calculus applied to finance, Physica A, 320:548- 570 .

Nelsen, R.B. (2006) An Introduction to Copulas. Springer Series in Statistics.

NozARI, M., RAEI, S.M., JAhANGiRI, P. \& BAHRAMgiri, M. (2010) A comparison of heavy-tailed $\mathrm{VaR}$ estimates and Filtered Historical Simulation: Evidence from emerging markets, International review of Business Research Papers, 6(4): 347-359.

PaOlella, M. (2007) Intermediate Probability: A Computational Approach. Wiley-Interscience, New York, NY, USA.

PichleR, S. \& SELITSCH, K. (1999) A comparison of analytical VaR methodologies for portfolios that include options. Proceedings of 8th Symposium on Finance, Banking and Insurance, University of Karlsruhe, Germany.

PRITSKer, M. (1996) Evaluating Value-at-Risk methodologies: Accuracy versus computational time. Working paper, Wharton Financial Institutions Centre. http://ideas.repec.org/p/wop/pennin/9648.html.

Rahman, M., JiAn-PInG, Z. \& Yi-HANG, S. (2011) An application of copula theory in the estimation of Value at Risk in case of Bangladeshi stock markets, International Research Journal of Finance and Economics, 71:118-131.

RAO, C.R. (2001) Linear Statistical Inference and its Applications (2nd Edition). John Wiley \& Sons.

Rockafellar, R.T. \& URYASEV, S. (2000) Optimization of conditional Value-at-Risk, J. Risk, 2:2141.

Rouvinez, C. (1997) Going greek with VaR, Risk, 10(2):57-65.

SAK, H. \& HAKsÖZ, C. (2011) A copula-based simulation model for supply portfolio risk, The Journal of Operational Risk, 6(3):15-38.

SHAO, Q., WANG, H. \& YU, H. (2006) A calibrated scenario generation model for heavy-tailed risk factors, IMA J. Manage. Math., 17:289-303.

SHEIKH, A.Z. \& QIAO, H. (2010) Non-normality of market returns: a framework for asset allocation decision-making, Journal of Alternative Investments, 12(3):8-35.

Sornette, D., Simonetti, P. \& Andersen, J.V. (2000) $\phi^{q}$-field theory for portfolio optimization: "fat tails" and nonlinear correlations, Phys. Rep., 335(2):19-92. 
Stoyanov, S.V., Rachev, S.T., Racheva-Iotova, B. \& Fabozzi, F.J. (2011) Fattailed models for risk estimation, Working Paper Series in Economics, Karlsruhe Institute of Technology (KIT), Department of Economics and Business Engineering, 30:1-22. http://EconPapers.repec.org/RePEc:zbw:kitwps:30.

SzEGÖ, G. (2005) Measures of risk, Eur. J. Oper. Res., 163(1):5-19.

ZANGARI, P. (1996) A VaR methodology for portfolios that include options. RiskMetrics Monitor, first quarter 1996.

ZANGARI, P. (1996) An improved methodology for measuring VaR. RiskMetrics Monitor, second quarter 1996.

ZANGARI, P. (1996) How accurate is the Delta-Gamma methodology? RiskMetrics Monitor, third quarter 1996. 